This item was submitted to Loughborough's Research Repository by the author.

Items in Figshare are protected by copyright, with all rights reserved, unless otherwise indicated.

\title{
Optimal design of PID controllers using the QFT method
}

PLEASE CITE THE PUBLISHED VERSION

PUBLISHER

(C) IEEE

VERSION

AM (Accepted Manuscript)

LICENCE

CC BY-NC-ND 4.0

REPOSITORY RECORD

Zolotas, Argyrios C., and G.D. Halikias. 2019. "Optimal Design of PID Controllers Using the QFT Method". figshare. https://hdl.handle.net/2134/4327. 
This item was submitted to Loughborough's Institutional Repository (https://dspace.lboro.ac.uk/) by the author and is made available under the following Creative Commons Licence conditions.

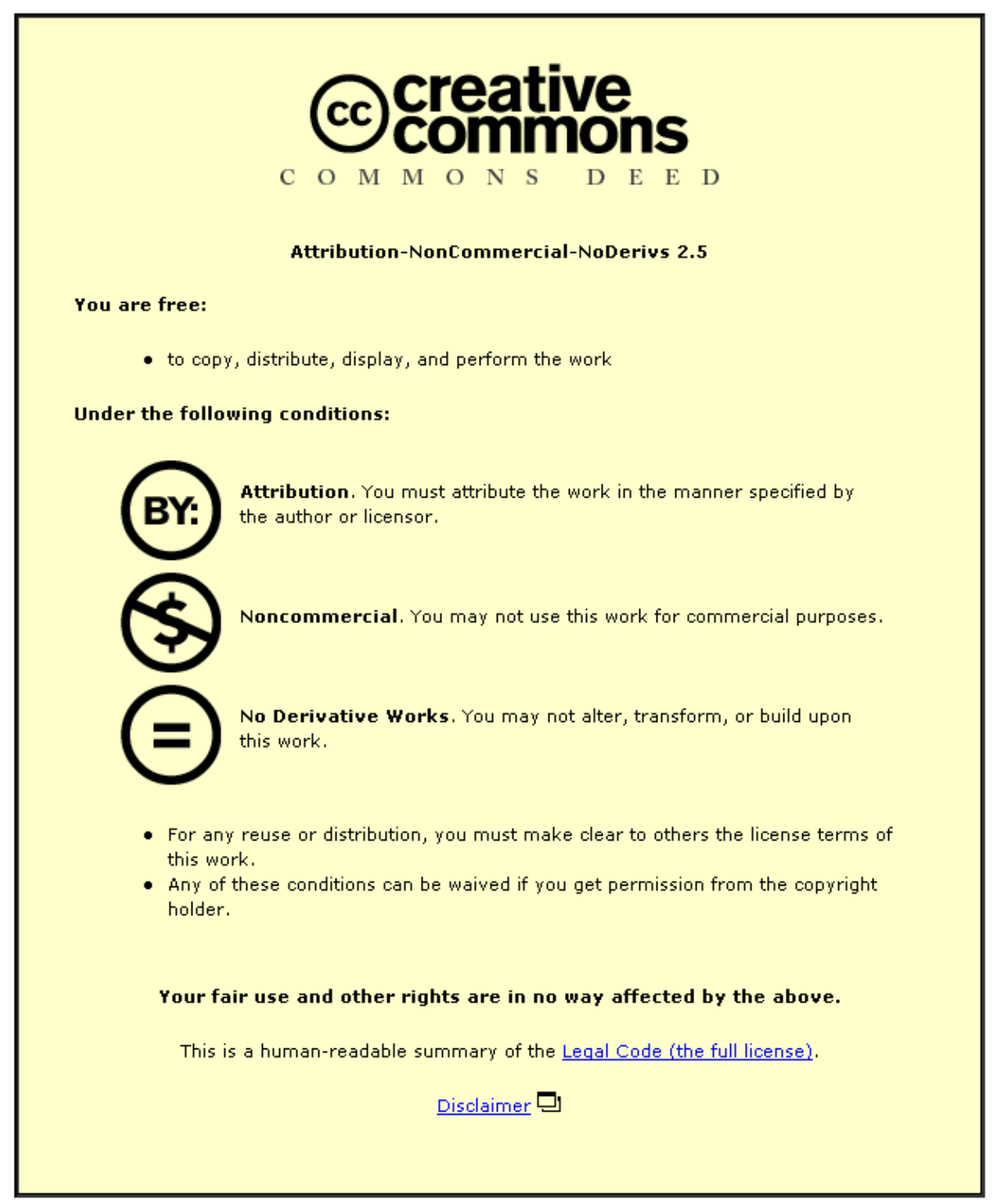

For the full text of this licence, please go to: http://creativecommons.org/licenses/by-nc-nd/2.5/ 


\title{
Optimal design of PID controllers using the QFT method
}

\author{
A. C. Zolotas ${ }^{1}$ and G. D. Halikias ${ }^{2}$
}

\begin{abstract}
An optimisation algorithm is proposed for designing PID controllers, which minimises the asymptotic open-loop gain of a system, subject to appropriate robuststability and performance QFT constraints. The algorithm is simple and can be used to automate the loop-shaping step of the QFT design procedure. The effectiveness of the method is illustrated with an example.
\end{abstract}

Keywords: QFT; PID control; Robust control.

\section{Introduction}

Many practical systems are characterised by high uncertainty which makes it difficult to maintain good stability-margins and performance properties for the closed-loop system. There are two general design methodologies for dealing with the effects of uncertainty: (i) Adaptive control, in which the parameters of the plant (or some other appropriate structure) are identified on-line and the information obtained is then used to "tune" the controller, and (ii) Robust control, which typically involves a "worst-case" design approach for a family of plants (representing the uncertainty) using a single fixed controller.

Quantitative Feedback Theory (QFT) is a robust-control method developed during the last two decades which deals with the effects of uncertainty systematically. It has been sucessfully applied to the design of both SISO and MIMO systems, while the theory has also been extended to the nonlinear and the time-varying case. In comparison to other optimisation-based robust control methods, QFT offers a number of advantages. These include, (i) the ability to assess quantitatively the "cost of feedback" [3], [4], [5], (ii) the ability to take into account phase information in the design process (which is lost if, e.g. singular values are used as the design parameters), and, (iii) the ability to provide "design transparency", i.e. clear tradeoff criteria between controller complexity and feasibility of the design objectives. Note that (iii) implies in practice that QFT often results in simple controllers which are easy to implement.

For the purposes of QFT, the feedback system is normally described by the twodegrees-of-freedom structure shown in Figure 1. In this diagram, $G(s)$ is the uncertain plant, $K(s)$ is the feedback controller and $F(s)$ is the pre-filter. The objective is to design

1 Department of Electronic and Electrical Engineering, Control Group, Loughborough University, Loughborough, LE11 3TU, UK

2 Department of Electronic and Electrical Engineering, University of Leeds, Leeds LS2 9JT, UK 
$K(s)$ and $F(s)$ so that the output signal $Y(s)$ tracks accurately the reference signal $R(s)$ and rejects the disturbance $D(s)$, despite the presence of uncertainty in $G(s)$.

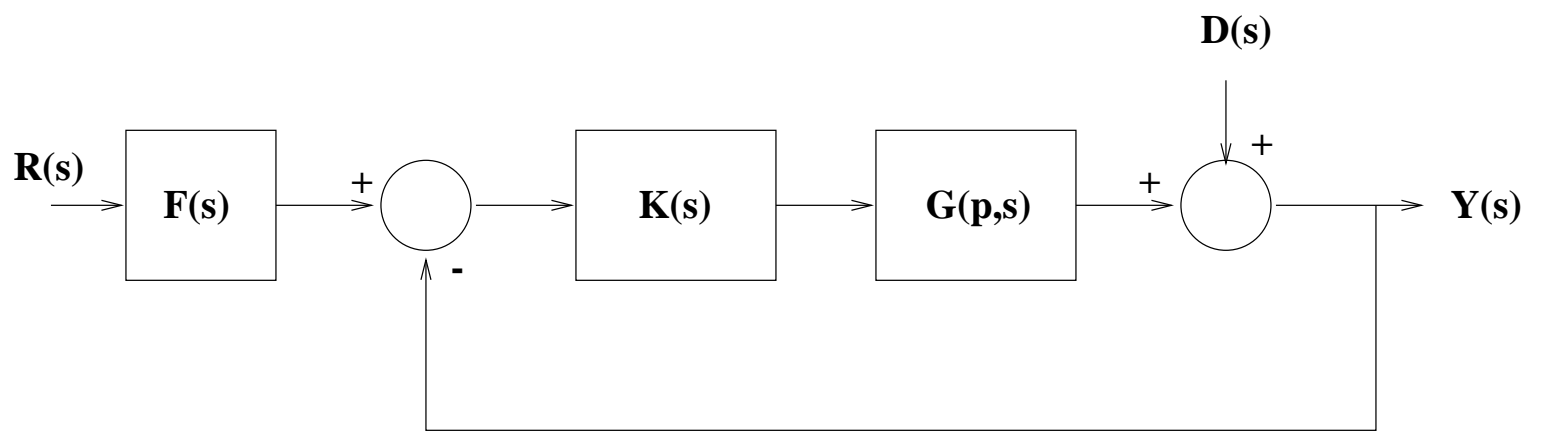

Figure 1: Two degree of freedom feedback system

The uncertain plant $G(s)$ is assumed to belong to a set, $G(s) \in\{G(p, s): p \in \mathcal{P}\}$, where $p$ is the vector of uncertain parameters; these are assumed to be fixed but unknown, and to take values in $\mathcal{P}$. The uncertain plant is first translated in the frequency domain (using a discrete grid of frequencies $\omega_{1}, \omega_{2}, \ldots, \omega_{N}$, typically chosen to cover adequately the system's bandwidth), resulting in $N$ "uncertainty templates" $\mathcal{G}_{i}=\left\{G\left(p, j \omega_{i}\right): p \in \mathcal{P}\right\}$, $i=1,2, \ldots, N$. The tracking specifications are given in the form of upper $\left(B_{u}\left(\omega_{i}\right)\right)$ and lower $\left(B_{l}\left(\omega_{i}\right)\right)$ bounds in the frequency domain, usually based on simple second-order models to represent appropriate underdamped and overdamped conditions. A similar procedure is followed to formulate the disturbance-rejection objectives of the design: In this case, the magnitude of the sensitivity function $\left|S\left(p, j \omega_{i}\right)\right|=\left|\left(1+G\left(p, j \omega_{i}\right) K\left(j \omega_{i}\right)\right)^{-1}\right|$ is required not to exceed an appropriate bound $D_{u}\left(\omega_{i}\right)$ for all $i=1,2, \ldots, N$ and every $p \in \mathcal{P}$. Note that the disturbance bounds are defined independently of the tracking bounds due to the presence of the prefilter, $F(s)$.

Next, the tracking and disturbance-rejection specifications are translated to certain conditions on the nominal open-loop frequency response $L_{o}(j \omega)=G_{o}(j \omega) K(j \omega)$ where $G_{o}(j \omega)$ denotes the nominal plant, defined for any $p \in \mathcal{P}$. Consider first the tracking bounds. For each frequency $\omega_{i}, i=1,2, \ldots, N$, the tracking specifications will be satisfied if and only if,

$$
\max _{p \in \mathcal{P}} \Delta\left|\frac{G\left(p, j \omega_{i}\right) K\left(j \omega_{i}\right)}{1+G\left(p, j \omega_{i}\right) K\left(j \omega_{i}\right)}\right|_{\mathrm{db}} \leq \delta\left(\omega_{i}\right):=\left.B_{u}\left(\omega_{i}\right)\right|_{\mathrm{db}}-\left.B_{l}\left(\omega_{i}\right)\right|_{\mathrm{db}}
$$

i.e. if and only if the maximum variation in the closed-loop gain as $p$ varies over the set $\mathcal{P}$, at each frequency $\omega_{i}$ does not exceed the maximum allowable spread in the specifications $\delta\left(\omega_{i}\right)$. This is because, (i) the uncertainty associated with $K$ is assumed negligible, and (ii) the actual closed-loop gain can always be adjusted to its required level at each frequency via the scaling action of the prefilter. For each frequency $\omega_{i}$, the open-loop gain (for each open-loop phase $\phi$ ) at which condition (1) is met with equality, defines the so-called "Horowitz template" $h\left(\omega_{i}, \phi\right)$; this is the minimum open-loop gain necessary to achieve 
the specified robust tracking specifications at frequency $\omega_{i}$. In practice, each "Horowitz template" is calculated via a bisection algorithm over a phase grid, and the equality condition in (1) is satisfied within a given tolerance. In total, we have $N$ Horowitz templates, one for each frequency of interest $\omega_{i}$. A similar procedure will result in $N$ robust disturbance-rejection contours, $d_{i}\left(\omega_{i}, \phi\right), i=1,2, \ldots, N$, which define the minimum open-loop gain required to achieve robust disturbance rejection (i.e. disturbance rejection for every $p \in \mathcal{P}$ ). Clearly, to achieve robust tracking and robust disturbance-rejection simultaneously, the open-loop frequency response must satisfy,

$$
\left|L\left(j \omega_{i}\right)\right| \geq f_{i}\left(\omega_{i}, \phi\right):=\max \left\{h_{i}\left(\omega_{i}, \phi\right), d_{i}\left(\omega_{i}, \phi\right)\right\}
$$

for all $i=1,2, \ldots, N$, where the maximum in $(2)$ is calculated pointwise in $\phi=\arg L\left(j \omega_{i}\right)$. The contours $f_{i}\left(\omega_{i}, \phi\right)$ will be referred to as the robust-performance bounds.

In addition to robust-performance objectives, the closed-loop system should also be robustly stable, i.e. the closed-loop transfer function $F(s) G(p, s) K(s)(1+G(p, s) K(s))^{-1}$ should be stable for every $p \in \mathcal{P}$. Since $F(s)$ will be designed stable, robust stability can be inferred from the number of encirclements around the -1 point by the openloop frequency response $L(p, j \omega)=G(p, j \omega) K(j \omega), p \in \mathcal{P}$. In practice, a more severe constraint is imposed on $L(p, j \omega)$ : To establish a minimum amount of damping for the (nominal) closed-loop system, the nominal open-loop response is constrained not to enter an $M$-circle of an appropriate value. Under the assumption of parametric uncertainty ${ }^{1}$ in the plant, the uncertainty templates of $G_{o}(j \omega)$ at high frequencies approach a vertical line in the Nichols chart. Hence, to ensure that the specified minimum amount of damping is maintained at high frequencies despite the presence of uncertainty, the $M$ circle is translated downwards by a specific amount, resulting in the so-called "universal high frequency boundary", which should not be penetrated by the nominal open-loop response [4]. This contour will be denoted by $\mathcal{B}$ in the sequel.

\section{Optimal Design of PID controllers}

After constructing the contours $f_{i}\left(\omega_{i}, \phi\right)$ and $\mathcal{B}$ on the Nichols' chart, QFT normally proceeds with the design of the feedback controller $K(s)$. This normally involves frequencyshaping of the nominal open-loop frequency response $L_{o}(j \omega)$, so that it does not penetrate the the $\mathcal{B}$ contour and $\left|L_{o}\left(j \omega_{i}\right)\right|$ lie on or above the robust-performace bounds $f_{i}\left(j \omega_{i}, \phi\right)$ for each $\omega_{i}$. At this stage, the designer normally follows a phase-lead/lag compensation approach which involves a considerable trial-and-error element, and can be cumbersome, especially if the number of specified frequencies is large and the specifications are tight. If the specifications can not be achieved, the design objectives are assumed to be infeasible. In this case, the specifications are normally relaxed and the design is repeated. If

\footnotetext{
1 The method can be modified to also take into account unstructured high frequency uncertainty.
} 
the specifications are feasible, the best design is considered to be the one for which the specifications are met as tightly as possible. This is in order to avoid the possibility of "overdesigning" the system using unnecessarily large gains/bandwidth, which can result in measurement noise amplification and potential instability due to parasitics and highfrequency unmodelled dynamics [3], [4]. A compromise between controller complexity and a "tight" design has to be made in many cases.

In this section we present a simple algorithm for designing PID controllers which are optimal in the QFT sense. PID (or "three-term") controllers are widely used in industry because they are simple and easy to tune. Our algorithm can be used to provide an adequate QFT design, or as the first step for designing a more complex controller [1]. In addition, the algorithm can easily tackle a large number of constraints and can be also be applied to multivariable systems, using the standard QFT approach [7], [6].

A PID controller has a transfer function,

$$
K_{\text {pid }}(s)=k_{p}+\frac{k_{i}}{s}+k_{d} s
$$

and is therefore completely defined by the three terms $k_{p}$ (proportional gain), $k_{i}$ (integral gain) and $k_{d}$ (derivative gain). Its frequency response is

$$
K_{\text {pid }}(j \omega)=k_{p}-j \frac{k_{i}}{\omega}+j k_{d} \omega
$$

Suppose that $K_{\text {pid }}(s)$ is used in cascade with an uncertain plant $G(p, s)$. Then, the nominal open-loop system has frequency response $L_{o}(j \omega)=G_{o}(j \omega) K_{\text {pid }}(j \omega)$, where $G_{o}(s)$ denotes the nominal plant. Thus, the asymptotic gain of the nominal open loop system is given by,

$$
\lim _{\omega \rightarrow \infty}\left|G_{o}(j \omega)\left(k_{p}-j \frac{k_{i}}{\omega}+j k_{d} \omega\right)\right|
$$

Suppose that the asymptotic gain of the nominal plant is $\left|G_{o}(j \omega)\right| \sim A \omega^{-p}$ where the pole/zero excess of the nominal plant $p$ is at least equal to 2 . Then the asymptotic gain of the nominal open loop is $\left|L_{o}(j \omega)\right| \sim A\left|k_{d}\right| \omega^{-p+1}$. Since $A$ and $p$ are fixed, the nominal open-loop gain at high frequencies is minimised by minimising $\left|k_{d}\right|$. This objective is consistent with the aims of QFT theory outlined previously.

To design an optimal PID controller, consistent with the requirements of QFT, we formulate the following optimisation problem:

Minimise $\left|k_{d}\right|$ subject to the constraints:

1. $\left|L_{o}\left(j \omega_{i}\right)\right| \geq f_{i}\left(\omega_{i}, \phi\right)$ for all $i=1,2, \ldots, N$ where $\phi:=\arg L_{o}\left(j \omega_{i}\right)$.

2. $L_{o}\left(j \omega_{i}\right) \notin \mathcal{B}$ for all $i=1,2, \ldots, N$.

Since $\mathcal{B}$ is a closed contour which is defined for a range of phases only, it is always possible to combine constraints (1) and (2) for each $i=1,2, \ldots, N$ into a single constraint 
as long as $f_{i}\left(\omega_{i}, \phi_{i}\right)$ intersects $\mathcal{B}$ or lies entirely above it, by calculating the point-wise maximum of the two contours in the common phase range. This is almost always the case in practice, since robust performance objectives are almost never associated with frequencies significantly exceeding the closed-loop bandwidth. The (unlikely) case that a performance bound lies below the $\mathcal{B}$ contour can also be accomodated in our algorithm via an additional checking condition. To simplify the presentation, however, we will assume in the sequel that this does not occur, and the combined contours will be denoted by $\tilde{f}_{i}\left(\omega_{i}, \phi_{i}\right)$. The optimisation problem, therefore, takes the form: Minimise $\left|k_{d}\right|$ subject to $\left|L\left(j \omega_{i}\right)\right| \geq \tilde{f}_{i}\left(\omega_{i}, \phi\right)$ for all $i=1,2, \ldots, N$.

The magnitude (linear) and phase of $K_{\text {pid }}(j \omega)$ are given by,

$$
\left|K_{\text {pid }}(j \omega)\right|=\sqrt{k_{p}^{2}+\left(k_{d} \omega-\frac{k_{i}}{\omega}\right)^{2}}, \quad \arg \left(K_{\text {pid }}(j \omega)\right):=\psi(\omega)=\tan ^{-1}\left(\frac{k_{d} \omega-\frac{k_{i}}{\omega}}{k_{p}}\right)
$$

respectively. Now suppose that we fix the phase of $K_{\text {pid }}$ at two distinct frequencies $\omega_{i}$ and $\omega_{j}$, i.e. $\psi\left(j \omega_{i}\right)=\psi_{i}$ and $\psi\left(j \omega_{j}\right)=\psi_{j}$. Then,

$$
\psi\left(j \omega_{i}\right)=\psi_{i}=\tan ^{-1}\left(\frac{k_{d} \omega_{i}-\frac{k_{i}}{\omega_{i}}}{k_{p}}\right)
$$

which implies that

$$
k_{d}-\frac{k_{i}}{\omega_{i}^{2}}-\frac{k_{p} \tan \left(\psi_{i}\right)}{\omega_{i}}=0
$$

Similarly,

$$
k_{d}-\frac{k_{i}}{\omega_{j}^{2}}-\frac{k_{p} \tan \left(\psi_{j}\right)}{\omega_{j}}=0
$$

Equations (8) and (9) can be arranged in matrix form as:

$$
\left(\begin{array}{ccc}
1 & -\frac{1}{\omega_{i}^{2}} & -\frac{\tan \left(\psi_{i}\right)}{\omega_{i}} \\
1 & -\frac{1}{\omega_{j}^{2}} & -\frac{\tan \left(\psi_{j}\right)}{\omega_{j}}
\end{array}\right)\left(\begin{array}{c}
k_{d} \\
k_{i} \\
k_{p}
\end{array}\right)=0
$$

Denote the $2 \times 3$ matrix in equation (10) by $A\left(\psi_{i}, \psi_{j}\right)$. Clearly, $\operatorname{Rank}\left(A\left(\psi_{i}, \psi_{j}\right)\right)=2$ since $\omega_{i} \neq \omega_{j}$. Therefore, the kernel of $A\left(\psi_{i}, \psi_{j}\right)$ is a one-dimensional subspace of $\mathcal{R}^{3}$, which implies that the controller gains $k_{d}, k_{i}$ and $k_{p}$ are fixed up to scaling. Numerically, the kernel of $A\left(\psi_{i}, \psi_{j}\right)$ can be calculated easily using the Singular Value Decomposition.

Applying the Singular Value Decomposition to $A\left(\psi_{i}, \psi_{j}\right)$ gives:

$$
A\left(\psi_{i}, \psi_{j}\right)=\left(\begin{array}{cc}
U_{1} & U_{2}
\end{array}\right)\left(\begin{array}{ccc}
\sigma_{1} & 0 & 0 \\
0 & \sigma_{2} & 0
\end{array}\right)\left(\begin{array}{c}
V_{1}^{T} \\
V_{2}^{T}
\end{array}\right)
$$

where $V_{1}^{T}$ is a $2 \times 3$ matrix. Here $V_{2}$ spans the kernel of $A\left(\psi_{i}, \psi_{j}\right)$. Write $V_{2}^{T}=\left[V_{21} V_{22} V_{23}\right]$. Then,

$$
\left(\begin{array}{c}
k_{d} \\
k_{i} \\
k_{p}
\end{array}\right)=\lambda\left(\begin{array}{l}
V_{21} \\
V_{22} \\
V_{23}
\end{array}\right)
$$


where $\lambda$ is an arbitrary real constant. Using equation (6), the gain and phase of $K_{\text {pid }}(s)$ may be written as

$$
\left|K_{\text {pid }}(j \omega)\right|=|\lambda| \sqrt{V_{23}^{2}+\left(V_{21} \omega-\frac{V_{22}}{\omega}\right)^{2}}, \quad \psi(\omega)=\tan ^{-1}\left(\frac{V_{21} \omega-\frac{V_{22}}{\omega}}{V_{23}}\right)
$$

Note that equation (13) implies that fixing the phase of $K_{\text {pid }}(j \omega)$ at two frequencies, fixes the phase of $K_{\text {pid }}(j \omega)$ at any frequency $\omega$, and thus also the phase of $L_{o}(j \omega)$. In this case, minimising $\left|k_{d}\right|$ is equivalent to minimising $\left|\lambda V_{21}\right|$.

Under the constraint that $\psi\left(\omega_{i}\right)=\psi_{i}$ and $\psi\left(\omega_{j}\right)=\psi_{j}$, the QFT constraints are satisfied if and only if

$$
\left|L_{o}\left(j \omega_{k}\right)\right|_{\mathrm{db}} \geq \tilde{f}_{k}\left(\omega_{k}, \phi_{k}\right)
$$

for all $k=1,2, \ldots, N$, where $\phi_{k}=\arg \left(L_{o}\left(j \omega_{k}\right)\right)$. Note that since the phase of $K_{\text {pid }}(j \omega)$ is fixed at every frequency $\omega, \phi_{k}$ is fixed and known for all $k=1,2, \ldots, N$. In fact,

$$
\phi_{k}=\arg \left(G_{o}\left(j \omega_{k}\right)\right)+\tan ^{-1}\left(\frac{V_{21} \omega_{k}-\frac{V_{22}}{\omega_{k}}}{V_{23}}\right)
$$

Since,

$$
\left|L_{o}\left(j \omega_{k}\right)\right|_{\mathrm{db}}=\left|G_{o}\left(j \omega_{k}\right)\right|_{d b}+\left|K_{\mathrm{pid}}\left(j \omega_{k}\right)\right|_{\mathrm{db}}
$$

equation (14) is equivalent to

$$
\left|K_{\mathrm{pid}}\left(j \omega_{k}\right)\right|_{\mathrm{db}} \geq \tilde{f}_{k}\left(\omega_{k}, \phi_{k}\right)-\left|G_{o}\left(j \omega_{k}\right)\right|_{\mathrm{db}}
$$

for all $k=1,2, \ldots N$. Substituting from (13) shows that this is equivalent to

$$
20 \log _{10}|\lambda| \geq \max _{k=1,2, \ldots, N}\left(\tilde{f}_{k}\left(\omega_{k}, \psi_{k}\right)-\left|G_{o}\left(j \omega_{k}\right)\right|_{\mathrm{db}}-10 \log _{10}\left(V_{23}^{2}+\left(V_{21} \omega_{k}-\frac{V_{22}}{\omega_{k}}\right)^{2}\right)\right)
$$

or $|\lambda| \geq 10^{\frac{\beta}{20}}$ where we have defined,

$$
\beta=\max _{k \in\{1,2, \ldots N\}}\left(\tilde{f}_{k}\left(\omega_{k}, \phi_{k}\right)-\left|G_{o}\left(j \omega_{k}\right)\right|_{\mathrm{db}}-10 \log _{10}\left(V_{23}^{2}+\left(V_{21} \omega_{k}-\frac{V_{22}}{\omega_{k}}\right)^{2}\right)\right)
$$

Multiplying by $\left|V_{21}\right|$ and noting that $\left|k_{d}\right|=\left|\lambda V_{21}\right|$, implies that $\left|k_{d}\right| \geq\left|V_{21}\right| 10^{\frac{\beta}{20}}$. Hence, provided that the phase of $K_{\text {pid }}$ is fixed as $\arg \left(K_{\text {pid }}\left(j \omega_{i}\right)\right)=\psi_{i}$ and $\arg \left(K_{\text {pid }}\left(j \omega_{j}\right)\right)=\psi_{j}$, the minimum value of $\left|k_{d}\right|$ which achieves the robust-performance constraints is given by $\left|k_{d}^{*}\right|=\left|V_{21}^{i, j} 10^{\frac{\beta_{i, j}}{20}}\right|$, where the additional indexes $(i, j)$ introduced in $V_{21}$ and $\beta$ emphasise the dependence of these variables on $\left(\omega_{i}, \omega_{j}\right)$ and $\left(\psi_{i}, \psi_{j}\right)$. We can now formulate the following algorithm for solving the optimisation problem. In this algorithm, the gains $k_{i}, k_{d}$ and $k_{p}$ have been further constrained to be non-negative. This assumption can be removed, if desired, with minor modifications to the algorithm.

Algorithm 1: Given the plant's uncertainty templates $\mathcal{G}_{i}$, a nominal plant $G_{o}\left(j \omega_{i}\right) \in$ $\mathcal{G}_{i}$ and QFT contraint bounds $\tilde{f}_{i}\left(\omega_{i}, \phi\right)$, each defined at frequencies $\omega_{1}, \omega_{2}, \ldots, \omega_{N}$, the following algorithm calculates an optimal PID controller $K_{\text {pid }}^{*}(s)=k_{p}^{*}+\frac{k_{i}^{*}}{s}+k_{d}^{*} s$ with non-negative gains, if it exists: 
1. Obtain a phase array $\Phi$ by discretising the phase interval $\left(-360^{\circ}, 0^{\circ}\right)$.

2. Select any two distinct frequencies $\omega_{k}, \omega_{l} \in\left\{\omega_{1}, \omega_{2}, \ldots, \omega_{N}\right\}$.

3. Caclulate phase intervals $\Phi_{k}, \Phi_{l} \subseteq \Phi$ in which the nominal open-loop phase can vary at $\omega_{k}$ and $\omega_{l}$ if a PID controller is used. $\Phi_{k}\left(\Phi_{l}\right)$ contains the phases of $\Phi$ which lie within $\pm 90^{\circ}$ of the nominal plant phase $G_{o}\left(j \omega_{k}\right)\left(G_{o}\left(\omega_{l}\right)\right)$.

4. Initialise $m \times n$ arrays $K_{p}, K_{i}$ and $K_{d}$, where $m$ and $n$ are the sizes of $\Phi_{k}$ and $\Phi_{l}$ respectively.

5. For each $\left(\Phi_{k}(i), \Phi_{l}(j)\right) \in \Phi_{k} \times \Phi_{l}$

(a) Calculate $\psi_{i}=\Phi_{k}(i)-\arg \left(G_{o}\left(\omega_{k}\right)\right)$ and $\psi_{j}=\Phi_{l}(j)-\arg \left(G_{o}\left(\omega_{l}\right)\right)$.

(b) Calculate the singular value decomposition of $A\left(\psi_{i}, \psi_{k}\right)$ and the corresponding vector $V_{2}^{i, j}$ spanning its kernel.

(c) If any two elements of $V_{2}^{i, j}$ have opposite signs, set $K_{d}(i, j)=\infty$; Else, let $q$ be the (common) sign of the elements of $V_{2}^{i, j}$, calculate $\beta_{i, j}$ using equation (17), and set $\lambda=q 10^{\frac{\beta_{i, j}}{20}}, K_{d}(i, j)=\lambda V_{21}^{i, j}, K_{i}(i, j)=\lambda V_{22}^{i, j}$ and $K_{p}(i, j)=\lambda V_{23}^{i, j}$.

6. Calculate $\left(i^{*}, j^{*}\right) \in \operatorname{argmin}_{i, j} K_{d}(i, j)$. If $K_{d}\left(i^{*}, j^{*}\right)=\infty$, the constraints cannot be satisfied by a PID controller with non-negative gains; otherwise the optimal PID controller is $K^{*}(s)=K_{p}\left(i^{*}, j^{*}\right)+K_{i}\left(i^{*}, j^{*}\right) s^{-1}+K_{d}\left(i^{*}, j^{*}\right) s$.

\section{Remarks on Algorithm 1:}

- In step 1 the phase discretisation of the interval $\left(-360^{\circ}, 0^{\circ}\right)$ results in a grid of phases, typically equally-spaced. In practice, 50-100 phases are adequate. It is helpful to calculate the performance bounds over the same phase grid $(\Phi)$.

- In principle any two frequencies $\omega_{k}, \omega_{l}$ can be selected in step 2. Selecting these two frequencies reasonably far apart works well in practice.

- In step 3, the phase intervals $\Phi_{k}$ and $\Phi_{l}$ may be further restricted, if desired, to ensure that the nominal open-loop frequency response $L_{o}(j \omega)$ is shaped appropriately. This will also reduce the number of calculations in step 5 of the algorithm.

- The singular value decomposition in step 5(b) of the algorithm can be dispensed with altogether, by calculating $V_{2}^{i, j}$ analytically. This, however, increases the complexity of the algorithm and does not lead to any significant reduction in computation time.

- Step 5(c) of the algorithm requires the calculation of $\beta_{i, j}$ which in turn relies on the calculation of the performance bounds at phases specified by equation (15), which may not belong to the (discrete) phase array used to calculate the bounds (typically 
$\Phi)$. There is no difficulty, however, in estimating the performance bounds for these phases using the adjacent phase points, e.g. via linear interpolation. Alternatively, the performance bounds can be calculated exactly for the phases obtained from equation (15) via a bisection algorithm implemented between steps $5(\mathrm{a})$ and $5(\mathrm{c})$.

- The algorithm can be specialised, if desired, to calculate optimal PI of PD controllers. This requires only a one-dimensional search over a phase grid defined at a single frequency.

\section{Example}

In this section we illustrate our algorithm by means of a simple example. The uncertain plant is taken as

$$
G(s)=\frac{k a}{s^{2}+a s}
$$

in which the parameters $a$ and $k$ vary independently in the intervals $1 \leq a \leq 10$ and $1 \leq k \leq 10$ respectively. The nominal plant, $G_{o}(s)$, is taken to correspond to $a=1$ and $k=1$. The tracking specifications are defined as:

$$
\left|B_{l}\left(j \omega_{i}\right)\right| \leq\left|T\left(a, k, j \omega_{i}\right)\right| \leq\left|B_{u}\left(j \omega_{i}\right)\right|
$$

Here $T(a, k, s)=F(s) G(a, k, s) K(s)(1+G(a, k, s) K(s))^{-1}$ is the closed-loop transfer function and the lower and upper tracking bounds are defined as the magnitude frequency responses of the two systems

$$
B_{l}(s)=\frac{0.6585(s+30)}{(s+2+j 3.969)(s+2-j 3.969)}
$$

and

$$
B_{u}(s)=\frac{8400}{(s+3)(s+4)(s+10)(s+70)}
$$

at each $s=j \omega_{i}$. Note that the zero of $B_{l}(s)(s=-30)$ and the two fast poles in $B_{u}(s)$ ( $s=-10$ and $s=-70$ ) have been included to ensure that the the magnitude frequency responses of $B_{l}(s)$ and $B_{u}(s)$ diverge at high frequencies [2]. The frequencies of interest $\left\{\omega_{i}\right\}$ have been selected as $\omega_{1}=0.5, \omega_{2}=1, \omega_{3}=2, \omega_{4}=3, \omega_{5}=5, \omega_{6}=10, \omega_{7}=30$ and

$\omega_{8}=60 \mathrm{rads} / \mathrm{s}$. For simplicity, no disturbance-rejection objectives have been considered in this example.

The uncertainty templates of the plant at the eight frequencies of interest have been calculated numerically and are displayed in Figure 2. To reduce the number of calculations, each template has been replaced by its convex hull. This results in a minimal amount of conservativeness in this case. 


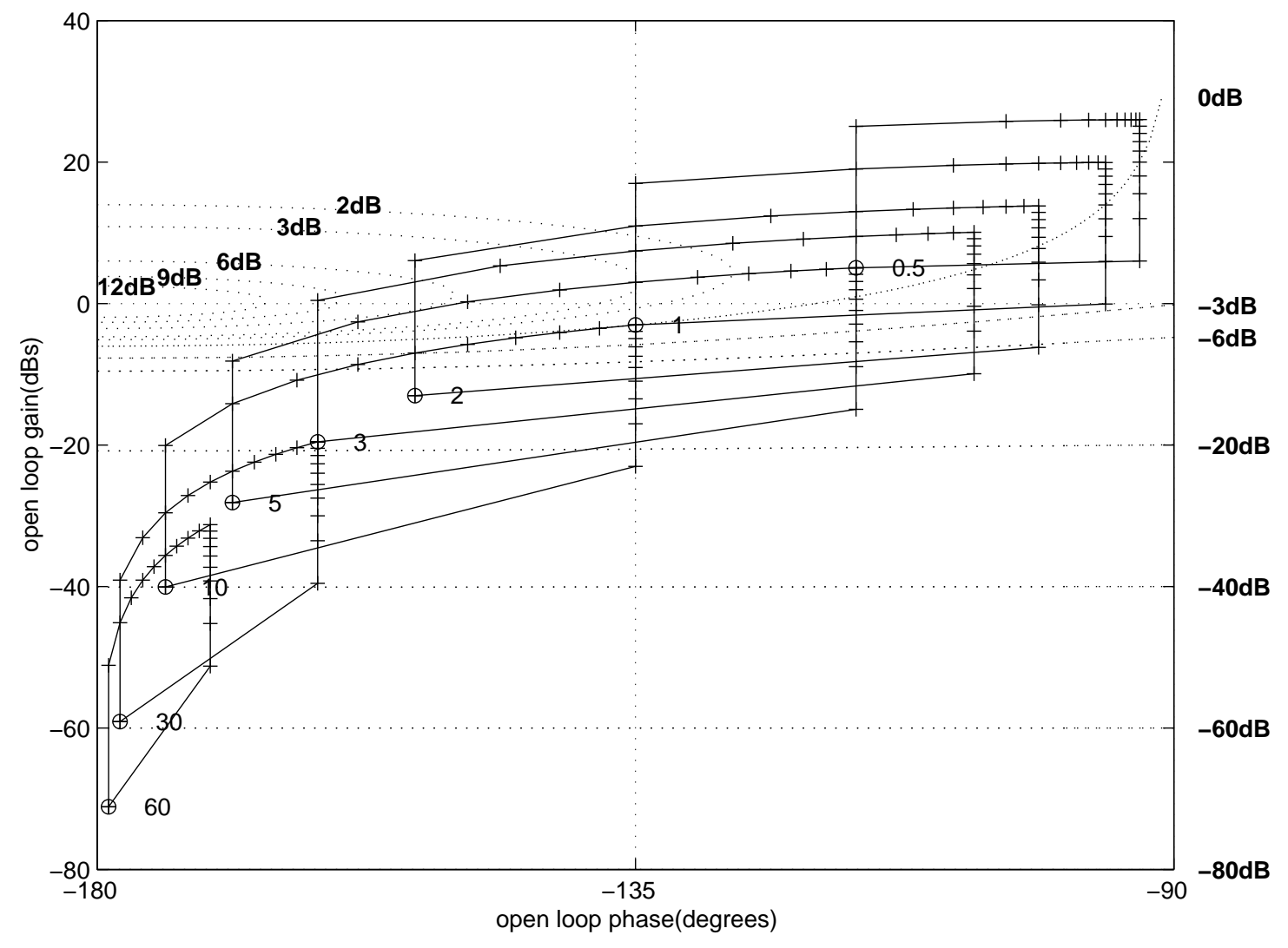

Figure 2: Uncertainty templates

Next, an optimal PID controller was designed following the procedure of Algorithm 1. The optimal controller was obtained as

$$
K_{\text {pid }}^{*}(s)=12.6+3.95 s+\frac{4.46}{s}
$$

Figure 3 shows the frequency response of the nominal plant (dashed line) and the nominal open loop (solid line) on the Nichols chart, together with the eight Horowitz templates and the $\mathcal{B}$-contour (corresponding to an $\mathrm{M}$ value of 1.2). The eight frequencies of interest are indicated by circles on the two frequency responses. The design meets the specifications, since the nominal frequency response does not penetrate the $\mathcal{B}$-contour and lies on or above the Horowitz templates at the eight frequencies of interest. As expected, one of the $L\left(j \omega_{i}\right)$ 's $\left(L\left(j \omega_{4}\right)\right)$ lies exactly on a bound (in this case the $\mathcal{B}$ contour).

Since the open-loop system has a pole-zero excess equal to one, its phase approaches $-90^{\circ}$ at high frequencies. There is no difficulty, however, in forcing the open loop response to approach the $-180^{\circ}$ phase line at high frequencies, if desired, by including a suitable $1+s \tau$ factor in the denominator of the controller derivative term. Choosing, for example, $\tau \ll \omega_{N}^{-1}$, has a minimal effect on the shape of $L_{o}(j \omega)$ for $\omega \leq \omega_{N}$. Alternatively, the PID controller may be assumed to be of the form

$$
K_{\text {pid }}^{\prime}(s)=k_{p}+\frac{k_{i}}{s}+\frac{k_{d} s}{1+s \tau}
$$




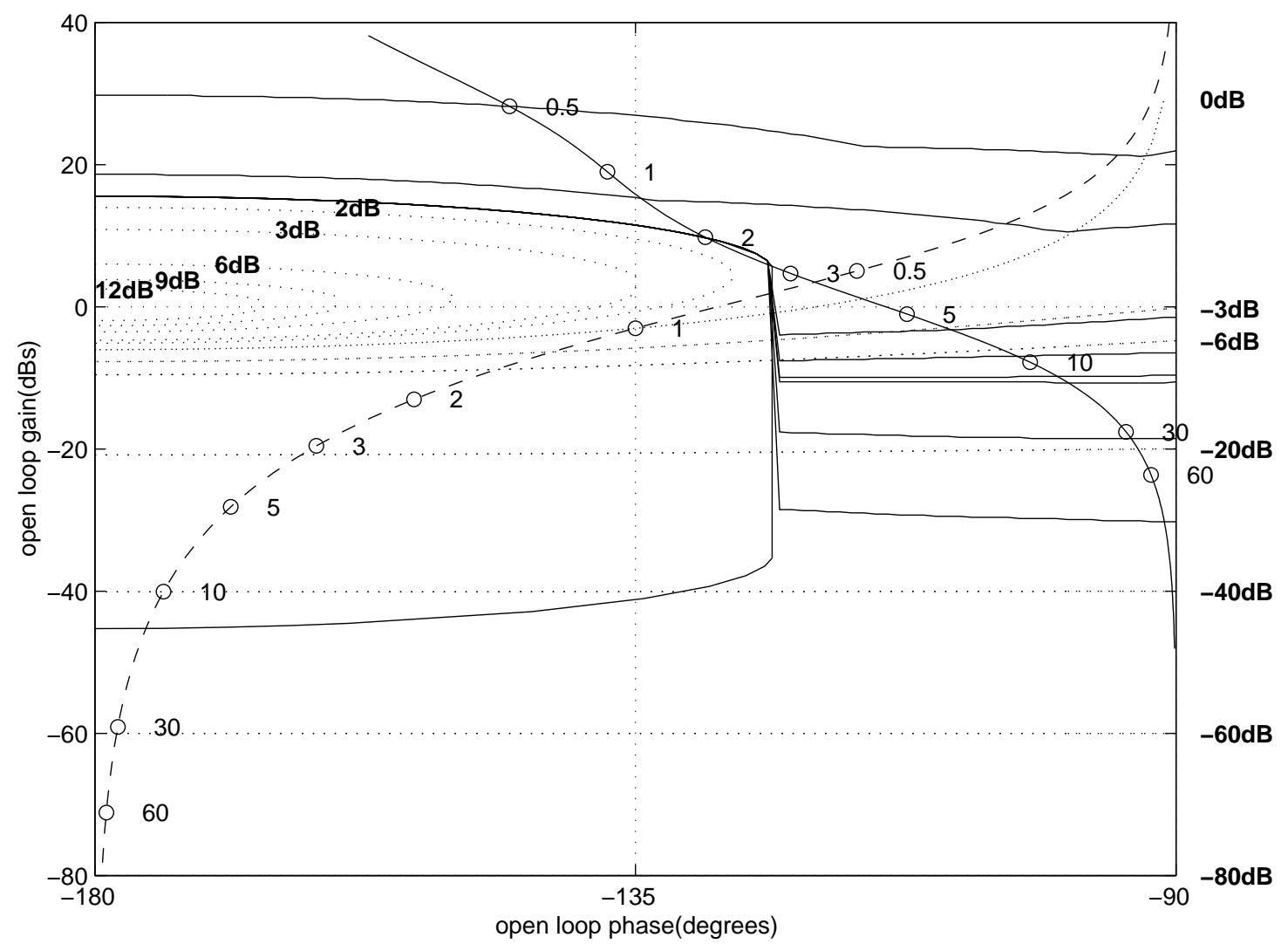

Figure 3: Nominal plant and nominal open-loop

( $\tau$ fixed) before solving the optimisation problem. Since in this case

$$
L_{o}^{\prime}(p, s):=K_{\text {pid }}^{\prime}(s) G(p, s)=\left(\left(k_{p}+k_{i} \tau\right)+\frac{k_{i}}{s}+\left(k_{d}+k_{p} \tau\right) s\right) \frac{G(p, s)}{1+s \tau}
$$

our algorithm can still be applied by redefining the uncertain plant as

$$
G^{\prime}(p, s)=\frac{G(p, s)}{1+s \tau}
$$

and optimising with respect to the new variables $k_{p}^{\prime}=k_{p}+k_{i} \tau, k_{i}^{\prime}=k_{i}$ and $k_{d}^{\prime}=k_{d}+k_{p} \tau$.

A second-order prefilter $F(s)$ (of dc gain equal to 1 and cut-off frequencies 3.5 and $7.5 \mathrm{rads} / \mathrm{s}$ ) was finally designed using the standard procedure [2]. Figure 4 shows the closed-loop frequency responses for a number of $(a, k)$ parameter combinations, together with the tracking bounds $\left|B_{l}(j \omega)\right|$ and $\left|B_{u}(j \omega)\right|$. Again, the eight frequencies of interest are marked by circles. Clearly, the specifications of the design are met, as expected from the characteristics of the open loop response in Figure 3.

\section{Conclusions}

An algorithm has been presented for designing optimal PID controllers for uncertain systems subject to QFT constraints. The algorithm is simple, easy to implement and can 


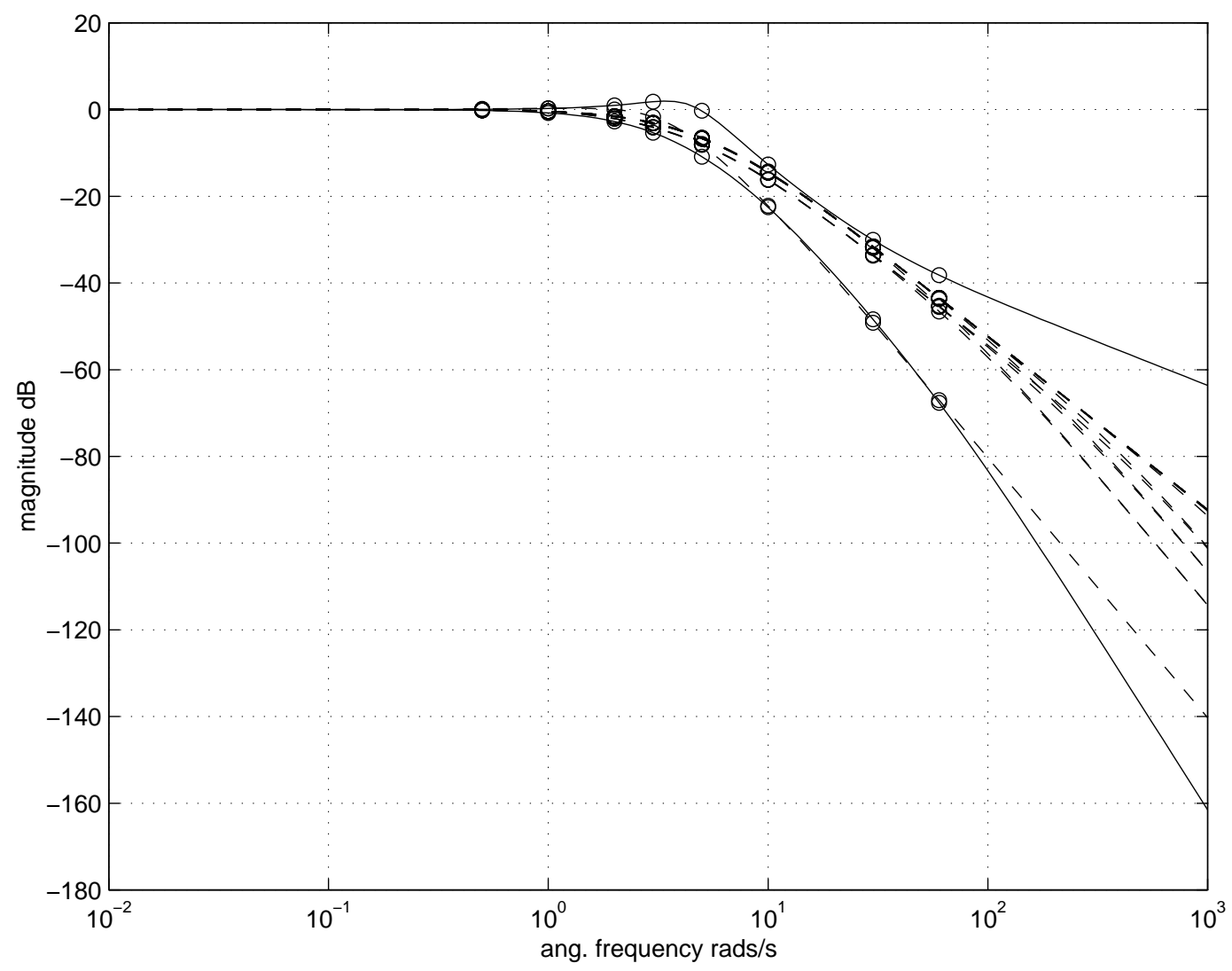

Figure 4: Closed-loop responses and bounds

be used to automate the loop-shaping step of the QFT design procedure. Although the algorithm has been presented for SISO systems, its extension to multivariable problems is possible using the standard QFT approach.

\section{Acknowledgement}

A. Zolotas would like to thank Mr Dimitriadis and Sevath ABE for providing him with the necessary computer equipment during the last phase of this work.

\section{References}

[1] BRYANT G.F. and HALIKIAS G.D., 1995, Optimal loop shaping for systems with large parameter uncertainty via linear programming. International Journal of Control, 62, 557-568.

[2] D'AZZO, J. and HOUPIS C., 1998, Feedback Control Systems Analysis and Synthesis (Prentice-Hall)

[3] HOROWITZ, I.M., 1973, Synthesis of linear Systems (Academic Press). 
[4] HOROWITZ, I.M. and SIDI, M., 1972, Synthesis of feedback systems with large plant ignorance for prescribed time-domain tolerances. International Journal of Control, 16, 287-309.

[5] HOROWITZ, I.M. and SIDI, M., 1978, Optimum synthesis of non-minimum phase systems with plant uncertainty, International Journal of Control, 27, 361-386.

[6] MACIEJOWSKI, J.M., 1989, Multivariable Feedback Design (Addison-Wesley).

[7] YANIV, O. and HOROWITZ, I.M., 1986, A Quantitative design method for MIMO linear feedback systems having uncertain plants. International Journal of Control, 43, 401-421. 